\title{
Effects of dual modified resistant indica rice starch on azoxymethane-induced incipient colon cancer in mice
}

\author{
HUAIBO YUAN* ${ }^{*}$ XIPING ZHU*, DEYI CHEN, WENJUAN WANG, SHAOHUA MENG and JUNHUI WANG \\ College of Biotechnology and Food Engineering, Hefei University of Technology, Hefei, Anhui 230009, P.R. China
}

Received January 9, 2015; Accepted February 19, 2016

DOI: $10.3892 /$ etm.2017.4172

\begin{abstract}
In this study, the effects of different doses of dual modification-treated (DMT) indica rice resistant starch (IR-RS) on azoxymethane (AOM)-induced early colon cancer in mice were investigated. The investigated factors included body weight, gastrointestinal emptying rate, the number and morphology of aberrant crypt foci (ACFs) and the specific expressions of adenomatous polyposis coli (APC), B-cell lymphoma 2 (Bcl-2), Bcl-2-associated X protein (Bax) and cytochrome $c$ genes. The results demonstrated that DMT IR-RS controlled the increase in the body weights of the mice, increased the gastrointestinal emptying rates and reduced the numbers of ACFs and aberrant crypts. Reverse transcription-polymerase chain reaction revealed that DMT IR-RS promoted the expression of APC, Bax and cytochrome $c$ and inhibited the expression of Bcl-2. These results demonstrate that a DMT IR-RS diet may induce apoptosis and has beneficial health effects in AOM-induced early colon cancer in mice.
\end{abstract}

\section{Introduction}

Colon cancer is the third most common cancer among women (after breast and lung cancers) and men (following prostate and lung cancers) and the third most common cancer-related cause of mortality globally, particularly in western and developed

Correspondence to: Professor Huaibo Yuan, College of Biotechnology and Food Engineering, Hefei University of Technology, 193 Tunxi Road, Hefei, Anhui 230009, P.R. China

E-mail: yuanhuaibo001@163.com

\section{${ }^{*}$ Contributed equally}

Abbreviations: IR-RS, indica rice resistant starch; DMT, dual modification-treated; LG, low-dose group; MG, middle-dose group; HG, high-dose group; PC, positive control; MC, model control; $\mathrm{NC}$, normal control; DW, distilled water; AOM, azoxymethane; CRC, colorectal cancer; ACF, aberrant crypt foci; RT-PCR, reverse transcription-polymerase chain reaction

Key words: resistant starch, gastrointestinal emptying rates, aberrant crypt foci, aberrant crypts, colon cancer nations (1,2). In addition, the incidence of colon cancer is higher in men than women and strongly increases with age (3). At present, colon cancer is one of the most frequently encountered malignant tumors, and the incidence of colon cancer ranks third among global gastrointestinal tumors (4). Economic development and improvements in living standards have caused marked changes in people's daily diets, and the morbidity and mortality of colon cancer have exhibited concurrent rapid increases in the last two decades. Morbidity and mortality are significantly higher in developed coastal areas than in the mainland. The incidence of colon cancer has strongly increased globally and is closely associated with elements of the so-called western lifestyle (5).

Colon cancer has long been associated with apoptosis and polygenic mutations $(4,6)$. The genetics of colon cancer have been actively researched in the final decades of the past century. The major genes identified as being associated with colon cancer are adenomatous polyposis coli (APC), B-cell lymphoma 2 (Bcl-2), Bcl-2-associated X protein (Bax) and cytochrome $c(7)$. Studies have shown that APC is a tumor suppressor gene. APC gene mutations can be detected in nearly all familial large adenomas and in $60-80 \%$ of sporadic colorectal cancers (CRCs) $(8,9)$. Apoptosis is regulated in part by the Bcl-2 gene, which promotes cell survival and lengthens cell life by hindering programmed cell death $(1,10)$. The expression of the Bcl-2 gene in colon cancer is higher than that in normal colonic mucosa and gradually increases as adenomas progress to early adenocarcinomas (11). Additionally, Bcl-2 can prevent the release of cytochrome $c$ from the mitochondria to the cytoplasm and thus inhibit apoptosis (12). Bax is a member of the Bcl-2 gene family whose products are associated with the Bcl-2-homologous proteins (13). The biological activities of Bcl-2 are antagonized by Bax. The main function of Bax is to accelerate cell apoptosis (14). Cytochrome $c$ is a water-soluble protein that is encoded by nuclear genes with a molecular weight of $12-13 \mathrm{kDa}$. The main function of cytochrome $c$ is to adjust mitochondrial energy metabolism (15). Studies have revealed that cytochrome $c$ also plays an important role in cell apoptosis via the transmission of the apoptosis signal and amplification of the regulation of apoptosis $(15,16)$.

The occurrence of colon cancer is attributable to a number of causes, but epidemiological studies have shown that dietary factors are important in the prevention of human colon cancer (17). Understanding the cause of colon cancer would undoubtedly contribute to better surveillance and early 
prevention and thus reduce cancer morbidity (18). Increasing the amount of fiber in the diet should reduce the incidence of cancers, particularly those of the colon and rectum $(19,20)$. Furthermore, resistant starch (RS) can reduce the incidence of colon cancer (21). Therefore, improved eating habits and greater dietary adjustments are the most economical and effective means of prevention and control.

$\mathrm{RS}$ is a starch, the chemical structure of which is different from that of fiber, and the properties of RS are similar to those of soluble fiber. RS is a new food ingredient and has a low glycemic index. RS is termed an anti-digested starch on the basis of the fraction of the starch that cannot be digested in the small intestine and is instead partially fermented in the large intestine to produce short-chain fatty acids and other products (22). In our previous study, RS was prepared from indica starch using a new method that combines $\alpha$-amylase, pullulanase and heat-moisture treatment. Indica rice resistant starch (IR-RS) products produce a mixture of B- and V-type $\mathrm{X}$-ray diffraction patterns and a crystallinity of $51.0 \%$ (23). In vivo experiments revealed that IR-RS is able to improve the symptoms associated with high blood sugar and the complications of diabetes in mice. Building upon our previous study, this study primarily focuses on the effect of IR-RS on azoxymethane (AOM)-induced colon cancer in mice and illuminates the mechanism of action of this effect. The results of this study should provide a scientific basis for colon cancer prevention and control measures, and be highly significant for the prevention of chronic diseases.

\section{Materials and methods}

Materials. Dual modification-treated (DMT) IR-RS was prepared according to the procedure outlined by Zhou et al (23). The RS content of the DMT IR-RS was $51.3 \%$. AOM was purchased from Sigma-Aldrich (St. Louis, MO, USA). Methylene blue, $10 \%$ buffered formalin and other chemicals and reagents were analytical grade.

Animals. One hundred male SCXK (su) 2011-001 mice (SPF mice that are free of specific microbes and parasites and so reduce experimental interference) were purchased from Changzhou Card Vince Laboratory Animal Co., Ltd. ('Changzhou, China). The experiments were approved by the ethical committee of the Animal Experiment Committee of China and conform to national guidelines on the care and use of laboratory animals (24).

AOM-induced colon cancer mice. During the experiments, the mice were housed in an approved laboratory animal facility and maintained under controlled temperature $\left(25^{\circ} \mathrm{C}\right)$ and lighting (12 h light/dark cycle) conditions for a 7-day adaptation period. AOM $(10 \mathrm{mg} / \mathrm{kg})$ diluted in normal saline was administered to the mice by intraperitoneal injection once per week for 2 consecutive weeks.

Experimental diets and general observation. Seven days after the first AOM injection, the model mice were placed in cages with 12 animals each and divided into the following five groups: Low-dose group (LG), middle-dose group (MG), high-dose group (HG), model control (MC) and normal control
(NC). The NC did not receive any treatment and other groups were treated with AOM. After the modeling and immediately after grouping, the mice in the LG, MG and HG groups were administered 2, 4 and $8 \mathrm{~g} / \mathrm{kg}$ DMT IR-RS aqueous solutions, respectively, once daily for 6 weeks by gavage. The mice in the MC and $\mathrm{NC}$ groups were given the same dose of distilled water by gavage. Each group had free access to food and water. The water bottles were filled twice daily. The drinking, diets and activities of the mice were observed daily, and the mice were weighed once per week.

Gastrointestinal emptying of mice. Gastrointestinal emptying was measured as previously described, with slight modifications $(25,26)$. Normal mice $(n=5)$ were fasted for $>12 \mathrm{~h}$ and had free access to water. The fasted mice were gavaged with different amounts of DMT IR-RS (2, 4 and $8 \mathrm{~g} / \mathrm{kg}$ ) or distilled water $(0.4 \mathrm{ml})$. Two hours later, each mouse was gavaged with $0.4 \mathrm{ml}$ blue ink. The mice were then sacrificed by cervical dislocation. The gut of each mouse was immediately exposed by laparotomy. The guts were expanded and a standard measuring tape was used to measure the distance that the blue ink had advanced in the gut (from the start of the gastric pylorus to the point at which the blue ink ended). Gastrointestinal emptying (\%) was calculated according to the following formula: Gastrointestinal emptying $(\%)=$ distance advanced/total length of the gut x 100 .

Observation of aberrant crypt foci (ACFs). After 8 weeks of gavage, all mice were sacrificed by cervical dislocation, and the colons were removed immediately, flushed with ice-cold normal saline to remove the intestinal contents, cut with scissors longitudinally, placed mucosal side up on strips of filter paper, and another filter paper was used to cover the mucosal surface. Finally, the colons were fixed in $10 \%$ buffered formalin for $24 \mathrm{~h}$. Each colon was then cut into $2-3 \mathrm{~cm}$ long strips. All segments were put into Petri dishes that contained $0.5 \%$ methylene blue solution for $2.5 \mathrm{~min}$ and then placed in another Petri dish with a buffer to wash away the excess dye. ACFs were immediately observed under a Gel Imaging System (BIS910; Beijing Dongsheng Innovative Biotechnology Co., Ltd., Beijing, China).

Reverse transcription-polymerase chain reaction (RT-PCR) for the detection of APC, Bax, Bcl-2 and cytochrome c mRNA. The primer sequences for APC, Bax, Bcl-2, cytochrome $c$ and GADPH were designed using Primer Premier 5 (Premier Biosoft International, Palo Alto, CA, USA) and synthesized by Shanghai Sangon Biological Engineering Technology \& Service Co. Ltd (Shanghai, China); the primer sequences are listed in Table I.

The total RNA of each mouse was extracted from the colon according to the method developed by Yuan et al (27) with slight modifications. The RNA extracts were placed in ultra-low temperature freezers $\left(-80^{\circ} \mathrm{C}\right)$ until use. cDNA was synthesized from total RNA with the Revert Aid First Strand cDNA Synthesis kit (Invitrogen; Thermo Fisher Scientific, Inc., Waltham, MA, USA) according to the manufacturer's protocol, and amplified by PCR. PCR was conducted for 30 cycles. Each amplification cycle consisted of $30 \mathrm{sec}$ at $93^{\circ} \mathrm{C}$ for denaturation, $30 \mathrm{sec}$ of cytochrome $c$ primer exposure at $59^{\circ} \mathrm{C}$, Bax 
Table I. Primer design for RT-PCR.

\begin{tabular}{llc}
\hline Gene & \multicolumn{1}{c}{ Primer sequence (5'-3') } & Product size (bp) \\
\hline Cytochrome $c$ & Forward: ACCAAATCTCCACGGTCTGTTC & 192 \\
& Reverse: GTCTGCCCTTTCTCCCTTCTTC \\
Bax & Forward: ACAGATCATGAAGACAGGGG \\
& Reverse: AAAGTAGAAGAGGGCAACCA \\
Bcl-2 & Forward: GTCACAGAGGGGCTACGAGT \\
& Reverse: GGGTCAGATGGACCACAGG \\
APC & Forward: GGAAGATTGGTTGTAAGTGAAAGGA & 298 \\
GAPDH & Reverse: CAAAAAGCAGAGTTAGAACAGGAGG & 212 \\
& Forward: CCCTTCATTGACCTCAACTAC & 244 \\
\hline
\end{tabular}

APC, adenomatous polyposis coli; Bcl-2, B-cell lymphoma 2; Bax, Bcl-2-associated X protein; RT-PCR, reverse transcription-polymerase chain reaction.

Table II. Body weight of each mouse.

\begin{tabular}{llllll}
\hline & \multicolumn{4}{c}{ Body weights $(\mathrm{g})$} \\
\cline { 2 - 6 } Group & Week 1 & Week 2 & Week 4 & Week 6 & Week 8 \\
\hline NC & $25.79 \pm 0.45$ & $31.56 \pm 0.15$ & $36.41 \pm 0.07$ & $38.98 \pm 0.35$ & $41.34 \pm 0.65$ \\
MC & $25.88 \pm 0.15$ & $27.91 \pm 0.28^{\mathrm{a}}$ & $35.61 \pm 0.20$ & $39.37 \pm 0.27$ & $40.76 \pm 0.51$ \\
LG & $25.14 \pm 0.33$ & $27.24 \pm 0.45^{\mathrm{a}}$ & $30.47 \pm 0.32^{\mathrm{a}}$ & $33.65 \pm 0.45$ & $38.36 \pm 0.08$ \\
MG & $25.52 \pm 0.22$ & $27.53 \pm 0.32^{\mathrm{a}}$ & $26.60 \pm 0.26^{\mathrm{b}}$ & $28.46 \pm 0.13^{\mathrm{a}}$ & $32.25 \pm 0.11$ \\
HG & $25.94 \pm 0.25$ & $26.95 \pm 0.35^{\mathrm{a}}$ & $26.75 \pm 0.06^{\mathrm{b}}$ & $27.52 \pm 0.09^{\mathrm{a}}$ & $28.22 \pm 0.65^{\mathrm{a}}$ \\
\hline
\end{tabular}

Each result represents the mean value \pm standard deviation $(\mathrm{n}=10) .{ }^{\mathrm{a}} \mathrm{P}<0.05$ and ${ }^{\mathrm{b}} \mathrm{P}<0.01$ vs. the $\mathrm{NC}$ group. $\mathrm{NC}$, normal control; $\mathrm{MC}$, model control; LG, low-dose group; MG, middle-dose group; HG, high-dose group.

primer exposure at $55^{\circ} \mathrm{C}, \mathrm{Bcl}-2$ primer exposure at $55^{\circ} \mathrm{C}, \mathrm{APC}$ primer exposure at $62^{\circ} \mathrm{C}$ or GADPH primer exposure at $55^{\circ} \mathrm{C}$ for primer annealing, and $1 \mathrm{~min}$ at $72^{\circ} \mathrm{C}$ for extension. All PCR products were mixed with $2 \mu \mathrm{l}$ goldview loading dye (Invitrogen; Thermo Fisher Scientific, Inc.) and subjected to electrophoresis using $1.5 \%$ agarose gels containing $0.5 \mu \mathrm{g} / \mathrm{ml}$ ethidium bromide (Invitrogen; Thermo Fisher Scientific, Inc.).

Statistical analysis. All experiments were repeated $\geq 10$ times. All experimental data are expressed as the mean \pm standard deviation. Differences between test subjects and model controls were evaluated using Student's t-tests. $\mathrm{P}<0.05$ was considered to indicate a statistically significant difference.

\section{Results and Discussion}

Observations of the daily activities and body weights of the mice. During the experiment, each group of mice exhibited normal eating, drinking and activity. The changes in body weight in each group of mice during the experiment were analyzed, and the results are summarized in Table II. The results revealed no significant differences $(\mathrm{P}>0.05)$ in initial body weight $(25.65 \pm 0.33 \mathrm{~g})$ prior to the $\mathrm{AOM}$ injections between the five experimental groups. After the AOM injections (week 2), the body weights of the mice in the NC group increased by $22.4 \%$, while the other four groups of mice gained less weight than did the normal group, and there were no significant difference between the four experimental groups. After 6 weeks of feeding with RS solution (LG, $2 \mathrm{~g} / \mathrm{kg}$; $\mathrm{MG}, 4 \mathrm{~g} / \mathrm{kg} ; \mathrm{HG}, 8 \mathrm{~g} / \mathrm{kg}$ ), the gains in body weight of the mice in the LG, MG and HG groups were lower than those of the $\mathrm{NC}$ group, and the weight gain of the HG group was significantly different from that of the $\mathrm{NC}$ group $(\mathrm{P}<0.05)$. The body weights of the MC, LG and MG groups increased by 57.5, 52.7 and $26.4 \%$, respectively, and there were no significant differences relative to the $\mathrm{NC}$ group $(\mathrm{P}>0.05)$.

The results revealed that the body weights of the $\mathrm{MC}$ group increased rapidly and were not significantly different $(\mathrm{P}>0.05)$ from those of the NC group at 8 weeks. This finding indicates that the inchoate colon cancer induced by AOM had no clear effect on the body weights of the mice. However, the body weight gains in the RS-treated groups exhibited a dose-dependent reduction, which indicates that RS effectively controlled the increases in the body weights of the mice. In addition, some differences were observed in the food intake of the mice in different treatment groups. The amount of 
Table III. Influence of RS on colonic ACFs in mice.

\begin{tabular}{lcccc}
\hline Group & ACF incidence & Number of ACFs & Number of aberrant crypts & Mean number of aberrant crypts \\
\hline NC & $0 / 16$ & 0 & 0 & 0 \\
MC & $16 / 16$ & $11 \pm 2$ & $32 \pm 5$ & $3.0 \pm 0.13$ \\
LG & $16 / 16$ & $9 \pm 1$ & $25 \pm 5$ & $2.9 \pm 0.45$ \\
MG & $16 / 16$ & $8 \pm 1^{\mathrm{a}}$ & $26 \pm 4$ & $3.2 \pm 0.25$ \\
HG & $16 / 16$ & $7 \pm 1^{\mathrm{b}}$ & $20 \pm 3^{\mathrm{b}}$ & $3.0 \pm 0.16$ \\
\hline
\end{tabular}

Values are presented as the mean value \pm the standard deviation $(\mathrm{n}=10)$. ${ }^{\mathrm{a}} \mathrm{P}<0.05$ and ${ }^{\mathrm{b}} \mathrm{P}<0.01$ vs. the MC group. RS, resistant starch; $\mathrm{ACF}$, aberrant crypt foci; NC, normal control; MC, model control; LG, low-dose group; MG, middle-dose group; HG, high-dose group.

food intake decreased in mice after feeding with the RS solution, and the $\mathrm{NC}$ and $\mathrm{MC}$ groups exhibited normal eating. RS does not readily degrade in the small intestine; thus, RS can reduce the postprandial glycemic response and food intake and increase satiety (28). RS can also be fermented to short-chain fatty acids by microorganisms in the colon (29). Following the rapid absorption of short-chain fatty acids by colorectal tissue, energy is stored and the osmotic pressure is reduced; short-chain fatty acids are important in maintaining the morphology and function of the normal colon and colonic epithelial cell function (30). Therefore, RS can reduce the intestinal $\mathrm{pH}$ and the quantity of carcinogens, and promote the absorption of trace elements.

Effects of RS on gastrointestinal emptying. Gastrointestinal emptying plays different and important roles in accommodating gastrointestinal function (31). As shown in Fig. 1, the gastrointestinal emptying rate of the normal mice increased following DMT IR-RS gavage. The gastrointestinal emptying rate of the high-dose group $(38.5 \pm 0.9 \%)$ was significantly different from that of the mice treated with distilled water only $(\mathrm{P}<0.01)$. Treatment with 2 or $4 \mathrm{~g} / \mathrm{kg}$ IR-RS did not markedly affect gastrointestinal emptying rates, which indicates that a certain amount of RS helps to improve gastrointestinal emptying and promote gastrointestinal motility. These results should aid the analysis of the role of RS in gastrointestinal motility and may provide a new strategy for future studies of colon cancer.

Effects of RS on ACFs in the colons of mice. Following the intraperitoneal injection of AOM, the incidence of ACFs in the mice was $100 \%$. The ACF configurations are presented in Fig. 2A. The ACF incidence in the NC group was $0 \%$, and the configuration is shown in Fig. 2B. The ACF incidence revealed that the colon cancer model was successful. In comparison with the NC group, the ACF characteristics of the other groups included comparatively large crypt foci, deep staining, oval cavity recesses and additional layers of epithelial cells.

Table III summarizes the effect of RS on the ACFs of the mice. The numbers of ACFs in the RS-treated groups exhibited a dose-dependent reduction compared with those in the MC group, while the MG and $\mathrm{HG}$ groups had significantly different numbers of ACFs from the MC group $(\mathrm{P}<0.05)$. In the RS-treated groups, the numbers of aberrant crypts in the LG group did not differ from those of the MG group ( $\mathrm{P}>0.05)$,

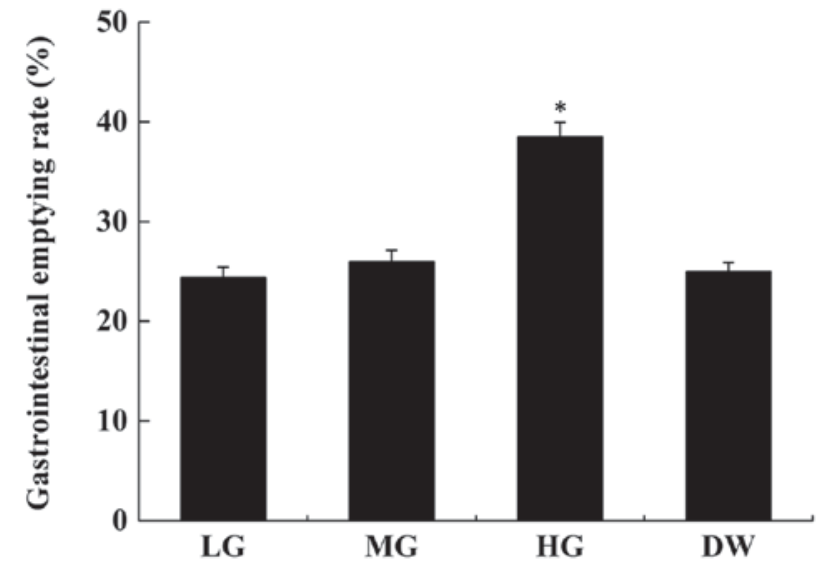

Figure 1. Influence of RS on gastrointestinal emptying rate. Results are expressed as the mean \pm standard deviation. ${ }^{*} \mathrm{P}<0.01$ vs. the DW group. LG, low-dose group; MG, middle-dose group; HG, high-dose group; DW, distilled water group.

and they were also not significantly different from those in the $\mathrm{MC}$ group. The number of aberrant crypts in the HG group was significantly different from that of the MC group $(\mathrm{P}<0.01)$. The average numbers of aberrant crypts in each ACF of the RS-treated groups were not dose-dependent, and the differences were not statistically significant. These results indicate that a specific diet of RS can effectively reduce the numbers of ACFs and aberrant crypts.

ACFs were first proposed by Bird et al (32) in 1987 and can be observed in CRC when viewing early colorectal mucosal lesions under an optical microscope. ACFs typically consist of one, several or even hundreds of abnormal aberrant crypts (33). The mechanism of the effect of RS on ACFs is mediated by $\mathrm{RS}$ inducing increases in diet and intestinal emptying rate, reducing fecal $\mathrm{pH}$, and increasing the levels of intestinal short chain fatty acids, which dilutes carcinogens, accelerates their excretion, and reduces their likelihood of coming into contact with the intestinal epithelium, and thereby suppresses the occurrence of ACFs (34).

Effect of RS on the expression of APC, Bax, cytochrome c and Bcl-2 genes in the colon. RT-PCR assays were used to detect the specific mRNA expression levels of APC, Bax, cytochrome $c$ and Bcl-2, and the results are shown in Fig. 3. GAPDH served as an internal reference for the calculation of the relative expression levels of the mRNAs. At 6 weeks of RS 

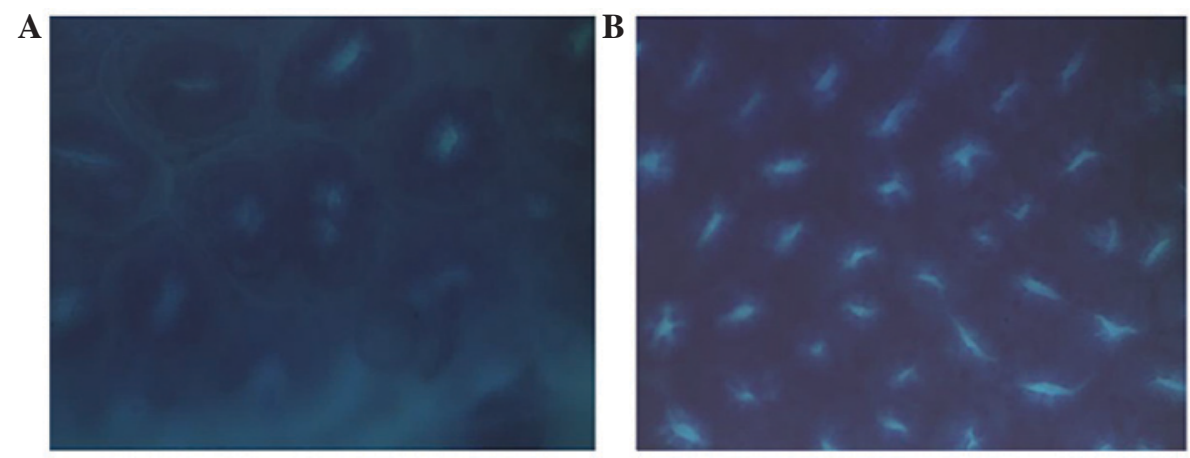

Figure 2. Shapes of the ACFs in the mice examined using light microscopy. (A) ACF configurations induced by the injection of azoxymethane, and (B) the NC group (methylene blue staining). The ACF incidence in the NC group was 0\%. Magnification, x100. ACF, aberrant crypt foci; NC, normal control.

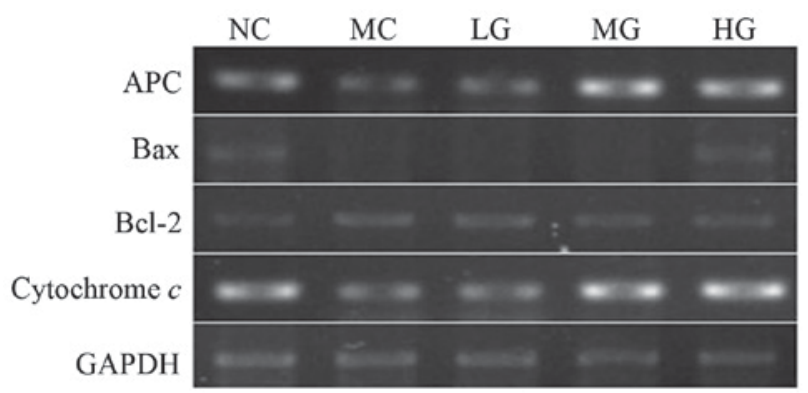

Figure 3. Expression of APC, Bax, cytochrome $c$ and Bcl-2 in the colon. Reverse transcription-polymerase chain reaction assays were used to detect the specific expression levels of APC, Bax, cytochrome $c$ and Bcl-2 genes. APC, adenomatous polyposis coli; Bcl-2, B-cell lymphoma 2; Bax, Bcl-2-associated X protein; NC, normal control; MC, model control; LG, low-dose group; MG, middle-dose group; HG, high-dose group.

gavage, the expression levels of the amplification products of $\mathrm{APC}, \mathrm{Bax}$ and cytochrome $c$ in the NC group were higher than those of the MC group. It was also observed that RS treatment promoted the expression of APC, Bax and cytochrome $c$ and that these increases exhibited a trend toward being dose-dependent. By contrast, the expression of the anti-apoptotic gene $\mathrm{Bcl}-2$ in the $\mathrm{NC}$ group was lower than that of the MC group. The results showed that RS treatment inhibited the expression of $\mathrm{Bcl}-2$, and these reductions exhibited a trend towards being dose-dependent.

The occurrence of CRC followed the adenoma to adenocarcinoma' law of development that was first proposed by Muto et al (35) in 1975. Fearon and Vogelstein (36) subsequently noted that the progression of 'adenoma to adenocarcinoma' involves changes in suppressor genes and oncogenes. Additionally, the mechanism of CRC also involves changes in signaling pathways. The occurrence of CRC is caused by changes in these genes and their long-term accumulation, and its mechanism is essentially a polygenic multi-step process. The APC gene is an important tumor suppressor gene in colon cancer and is located on human chromosome 5q21 (9). Deletions or mutations of the APC gene are closely associated with the occurrence of early CRC; $85 \%$ of colon cancers are accompanied by deletions and inactivation of the APC gene. The protein product of the APC gene typically combines with $\beta$-connexin protein ( $\beta$-catenin) to control excessive cell proliferation. When APC is mutated, its ability to regulate cell proliferation is reduced. Therefore, the APC- $\beta$-catenin pathway is considered to be an important therapeutic target for early colon cancer $(9,37)$. In the present study, it was found that the expression of APC in the MC group was lower than that in the NC group; however, after 6 weeks of gavage treatment, APC expression was higher than that of the MC group and increased in a dose-dependent manner. These results may be due to the ability of dietary RS to reduce the methylation of the APC gene, enabling APC and $\beta$-catenin to bind and thereby inhibit excessive cell proliferation.

The Bcl-2 family, which includes Bcl-2 and Bax genes, plays an important role in the regulation of apoptosis (38). In normal colonic mucosa, the Bcl-2 gene was found to be expressed in the mucosal crypts, and no cell apoptosis occurred, whereas the Bax gene was expressed in epithelial cells and promoted cell apoptosis. These findings indicate that these genes coordinate in normal mucosa to control cell proliferation and apoptosis so that the epithelial cells, which are produced by the mucosal crypts can migrate upward until death due to cellular aging (39). It has been found that Bcl-2 can antagonize the pro-apoptotic effect of Bax on cells and that the Bcl-2/Bax ratio is closely associated with apoptosis; indeed, this ratio is considered to be a molecular switch for the initiation of apoptosis (13). The present study found that the expression of the Bax gene in the $\mathrm{MC}$ group was weaker than that in the $\mathrm{NC}$ group, and that the expression of the Bcl-2 gene in the MC group was stronger than that in the $\mathrm{NC}$ group, in which the Bcl-2/Bax ratio was relatively high. However, after 6 weeks of gavage treatment, Bax gene expression increased, and $\mathrm{Bcl}-2$ gene expression decreased to produce a relatively low $\mathrm{Bcl}-2 / \mathrm{Bax}$ ratio. These results demonstrate that RS improved the abnormal expression of the Bcl-2 family of pro- and anti-apoptotic genes in the colon mucosa to bring the $\mathrm{Bcl}-2 / \mathrm{Bax}$ ratio close to that of the $\mathrm{MC}$ group and induce apoptosis.

Cytochrome $c$ is a water-soluble protein that regulates the energy metabolism of the mitochondria and also regulates apoptosis (15). Cytochrome $c$ plays a negative regulatory role and inhibits the growth of tumor cells in the process of tumor development. The mechanism of action of cytochrome $c$ is mediated by apoptotic signal conduction and amplification that results in the regulation of apoptosis (12). Cytochrome $c$ is closely associated with the Bcl-2 family. The Bcl-2 family can participate in the process of cytochrome $c$ release and the 
formation of an integral membrane protein. Bcl-2 stabilizes the mitochondrial membrane and suppresses the release and activation of cytochrome $c$ and caspase, which changes the oxidation-reduction reaction within the nucleus and ultimately inhibits apoptosis. It has been reported that the content of cytochrome $c$ in colorectal tissues adjacent to cancerous tissue is significantly higher than that in the cancerous tissues, which suggests that tumors may be associated with inhibition of the release of cytochrome $c$. In the present study, it was found that the expression of cytochrome $c$ mRNA in the MC group was weaker than that in the NC group. However, after 6 weeks of gavage treatment, cytochrome $c$ mRNA expression increased, and this result demonstrates that RS effectively increased the release of cytochrome $c$ from the colon mucosa cells. The results for APC, Bax, cytochrome $c$ and $\mathrm{Bcl}-2$ expression levels in the colon indicate that DMT IR-RS promoted the expression of APC, Bax and cytochrome $c$ and inhibited the expression of Bcl-2. These findings indicate that DMT IR-RS may induce colonic epithelial cell apoptosis and decrease the effects of colon cancer in mice.

In conclusion, the present study demonstrated that the DMT IR-RS product can induce apoptosis and has beneficial health effects in mice with AOM-induced early colon cancer. The body weight gains, gastrointestinal emptying rates and numbers of ACFs in the RS-treated groups decreased dose-dependently. In addition, DMT IR-RS diets may induce the occurrence of apoptosis and reduce the effects of colon cancer in mice. RS is difficult to digest in the small intestine and is fermented by microorganisms to increase the amounts of short-chain fatty acids, particularly butyric acid. Butyric acid can reduce carcinogen levels in the gut and inhibit tumor cells (40). In our future studies, we will examine how RS changes the intestinal microbial system to affect the generation and discharge of intestinal toxins and how toxins affect glucose metabolism in mice.

\section{Acknowledgements}

This study was funded by the National College Students Innovation Training Program (Grant No. 201210359058), the Department of Science and Technology, Anhui Province Natural Science Fund (Grant No. 1208085MC56) and The State Natural Science Fund (Grant No. 31370371).

\section{References}

1. John CR: Mechanisms of apoptosis. Am J Pathol 157: 1415-1430, 2000.

2. Homan SG, Steward BR and Armer JM: Public health and cooperative group partnership: A colorectal cancer intervention. Semin Oncol Nurs 30: 61-73, 2014.

3. Brenner H, Kloor M and Pox CP: Colorectal cancer. Lancet 383: 1490-1502, 2014.

4. Lv Q, Xing SY and Zhao ZH: Research status and prospect of colon cancer. Zhong Guo Shi Yan Zhen Duan Xue 13: 1134-1137, 2009.

5. Zheng S and Cai S: Colorectal cancer epidemiology and prevention study in China. Chinese-German J Clin Oncol 2: 72-75, 2003.

6. Tsukuda K, Tanino M, Soga H, Shimizu N and Shimizu K: A novel activating mutation of the K-ras gene in human primary colon adenocarcinoma. Biochem Biophys Res Commun 278: 653-658, 2000.

7. Tang N and Wang YY: Research progress of colon cancer related genes. Yi Xue Zong Shu 18: 207-209, 2012 (In Chinese).
8. Tang WZ, Gao F and Li W: APC mutation in sporadic colorectal cancer in China. Zhonghua Shi Yan Wai Ke Za Zhi 22: 1357-1359, 2005 (In Chinese).

9. Wu L, Qian YB and Zhu LX: Promoter methylation and mRNA expression of APC gene in hepatocellular carcinoma. Zhonghua Gan Dan Wai Ke Za Zhi 15: 378-381, 2007 (In Chinese).

10. Cory S and Adams JM: The Bcl 2 family: Regulators of the cellular life-or-death switch. Nat Rev Cancer 2: 647-656, 2002.

11. Zhu YS, Lu TY, Wang R, Huang M, Ma Q, Zhao L, Gao P, Lei X, Ni B, Lin J, et al: Functional conversion of Bcl-2 into a pro-apoptotic molecule to regulate mitochondrial cytochrome c. Sheng Ming Ke Xue 23: 1076-1080, 2011 (In Chinese).

12. Hockenbery D, Nuñez G, Milliman C, Schreiber RD and Korsmeyer SJ: Bcl-2 is a inner mitochorndrial membrane protein that blocks programmed cell death. Nature 348: 334-336, 1990.

13. Jia XD and Han C: Biomarkers in the studies on chemoprevention of colorectal cancer. Wei Sheng Yan Jiu 29: 109-111, 2000 (In Chinese).

14. Gong XZ and Liu W: The role of bcl-2 and bax gene in colon cancer. Qi Lu Yi Xue Za Zhi 17: 367-370, 2002 (In Chinese).

15. Chen NL, Chen H, Bai L, Zhang C and Zhao WH: Expression of cytochrome $c$ in colorectal cancer tissue and the ultramicro-structural change of mitochondria. Shiyong Yixue Zazhi 24: 4037-4039, 2008 (In Chinese).

16. Zhang J, Xu B, Cai WS and Li SH: The effect of Coixenolide extract on the histopathological changes induce by azoxymethane in rat colon cancer model. Zhongguo Yi Liao Qian Yan 6: 20-21, 2011 (In Chinese).

17. Rogers AE, Zeisel S and Groopman J: Diet and carcinogenesis. Carcinogenesis 14: 2205-2217, 1993.

18. Peh YC: Recent advances in colorectal cancer genetics and diagnostics. Crit Rev Oncol Hematol 69: 45-55, 2009.

19. Liu RP and Xu GF: Effects of resistant starch on colonic preneoplastic aberrant crypt foci in rats. Food Chem Toxicol 46: 2672-2679, 2008

20. Coleman LJ, Landström EK, Royle PJ, Bird AR and McIntosh GH: A diet containing alpha-cellulose and fish oil reduces aberrant crypt foci formation and modulates other possible markers for colon cancer risk in azoxymethane-treated rats. J Nutr 132: 2312-2318, 2002.

21. Brouns F, Kettlitz B and Arrigoni E: Resistant starch and 'the butyrate revolution'. Trends Food Sci Tech 13: 251-261, 2002.

22. Englyst HM, Kingman SM and Cummings JH: Classification and measurement of nutritionally important starch fractions. Eur J Clin Nutr 46 (Suppl 2): S33-S50, 1992.

23. Zhou Y, Meng S, Chen D, Zhu X and Yuan H: Structure characterization and hypoglycemic effects of dual modified resistant starch from indica rice starch. Carbohyd Polym 103: 81-86, 2014.

24. Institute of Laboratory Animal Resources (US). Committee on Care, Use of Laboratory Animals, and National Institutes of Health (US). Division of Research Resources: Guide for the care and use of laboratory animals. 8th edition. National Academies Press, Washington, DC, 2011.

25. Kim WK, Chung MK, Kang NE, K0im MH and Park OJ: Effect of resistant starch from corn or rice on glucose control, colonic events and blood lipid concentrations in streptozotocin-induced diabetic rats. J Nutr Biochem 14: 166-172, 2003.

26. Toden S, Bird AR, Topping DL and Conlon MA: Resistant starch prevents colonic DNA damage induced by high dietary cooked red meat or casein in rats. Cancer Biol Ther 5: 267-272, 2006.

27. Yuan HB, Gong ZB, Meng SH and He G: Hypoglycemic and hypolipidemic effects of a triterpenoid-rich extract from Euryale shell on streptozotocin-induced diabetic mice. Pharmazie 68: 227-231, 2013.

28. Zhao GH, Kan JQ, Li HJ and Chen Z: The research progress of resistant starch in food. Liang Shi Yu You Zhi 14: 37-40, 1999 (In Chinese).

29. Ma HB and Jia L: Progress in the stady of resisaant starch. Di Si Jun Yi Da Xue Ji Lin Jun Yi Xue Yuan Xue Bao 24: 174-176, 2002 (In Chinese).

30. Xu YJ, Fang RJ and Dai QZ: Physiological role of short-chain fatty acids in nutrition. Si Liao Yan Jiu 8: 26-28, 2007 (In Chinese).

31. Gaede P, Vedel P, Lavsen N, Jensen GV, Parving HH and Pedersen O: Multifactorial intervention and cardiovascular disease in patients with type 2 diabetes. New Engl J Med 348: 383-393, 2003.

32. Bird AR, Brown IL and Topping DL: Starches, resistant starches, the gut microflora and human health. Curr Issues Intest Microbiol 1: 25-37, 2000. 
33. Luebeck EG and Moolgavkar SH: Multistage carcinogenesis and the incidence of colorectal cancer. Proc Natl Acad Sci USA 99: 15095-15100, 2002.

34. Archer MC, Bruce WR, Chan CC, Corpet DE, Medline A Roncicci L, Stamp D and Zhang XM: Aberrant crypt foci and microadenoma as markers for colon cancer. Environ Health Perspect 98: 195-197, 1992.

35. Muto T, Bussey HJ and Morson BC: The evolution of cancer of the colon and rectum. Cancer 36: 2251-2270, 1975.

36. Fearon ER and Vogelstein B: Genetic model for colorectal tumorigenesis. Cell 61: 759-767, 1990.
37. Lee S, Lee HJ, Kim JH, Lee HS, Jang JJ and Kang GH: Aberrant $\mathrm{CpG}$ island hypermethylation along multistep hepatocarcinogenesis. Am J Pathol 163: 1371-1378, 2003.

38. Watson AJ, Merritt AJ, Jones LS, Askew JN, Anderson E, Becciolini A, Balzi M, Potten CS and Hickman JA: Evidence for reciprocity of bcl-2 and p53 expression in human colorectal adenomas and carcinomas. Br J Cancer 73: 889-895, 1996.

39. Yin XY, Oltval ZN and Korsmeyer SJ: BH1 and BH2 domains of bcl-2 are required for inhibition of apoptosis and heterodimerization with bax. Nature 369: 321-323, 1994.

40. Zhan Y: Butyric acid and tumor. Int J Cancer 30: 350-353, 2003. 